\title{
High-Order Schemes and Entropy Condition for Nonlinear Hyperbolic Systems of Conservation Laws
}

\author{
By J. P. Vila*
}

\begin{abstract}
A systematic procedure for constructing explicit, quasi second-order approximations to strictly hyperbolic systems of conservation laws is presented. These new schemes are obtained by correcting first-order schemes. We prove that limit solutions satisfy the entropy inequality. In the scalar case, we prove convergence to the unique entropy-satisfying solution if the initial scheme is Total Variation Decreasing (i.e., TVD) and consistent with the entropy condition. Finally, we slightly modify Harten's high-order schemes such that they obey the previous conditions and thus converge towards the "entropy" solution.
\end{abstract}

1. Introduction. We present here a systematic procedure for constructing explicit, quasi second-order approximations to hyperbolic systems of conservation laws. Other authors have recently dealt with high-order schemes: Majda and Osher [10], Harten [3], Le Roux and Quesseveur [9], Osher and Chakravarthy [11], but they have no result for systems, except in the case of time-continuous approximations ([10], [11]).

In Section 1 we recall basic useful features of numerical schemes for systems of conservation laws, in particular consistency with the system of conservation laws and its entropy condition (following ideas in Harten, Lax and Van-Leer [5]). In Section 2 we give examples of numerical schemes verifying consistency and study more precisely the scalar case with Total Variation Decreasing (TVD) schemes (see also Harten [3] and Tadmor [12], [13]). Section 3 is devoted to the description of our algorithm for constructing high-order schemes (see also Le Roux and Quesseveur [9]) and to the statement of our main result. We prove that limit solutions of our schemes are weak entropy solutions of the system of conservation laws. We also give more precise results concerning the scalar case and TVD high-order schemes. In Section 4 we examine a slight modification of Harten's high-order TVD schemes which enforces entropy inequalities for limit solutions. In the last section we present numerical experiments.

Received September 11, 1984; revised April 7, 1986 and September 17, 1986.

1980 Mathematics Subject Classification (1985 Revision). Primary 65M10, 35A40. 35L65.

* This work was carried out during the stay of the author at Ecole Polytechnique, Centre de Mathématiques Appliquées, 91128 Palaiseau Cedex, France and Ecole Nationale du Genie Rural des Eaux et des Forets, 19 avenue du Maine, 75732 Paris, Cedex, France. 


\section{Generalities.}

1.1. Systems of Conservation Laws. In this paper we consider numerical approximations to admissible weak solutions of the initial value problem (I.V.P.) for hyperbolic systems of conservation laws,

$$
\begin{cases}u_{, t}+f(u)_{. x}=0, & (x, t) \in \mathbf{R} \times \mathbf{R}_{+}, \\ u(x, 0)=u_{0}(x), & u \in R^{m} .\end{cases}
$$

The system is assumed to be strictly hyperbolic (i.e., $\lambda_{l}(u)$, the eigenvalues of $D f(u)$, are real and distinct),

$$
\lambda_{1}(u)<\cdots<\lambda_{l}(u)<\cdots<\lambda_{m}(u) .
$$

We consider systems of conservation laws that possess an entropy function $\eta(U)$ defined as follows:

$\eta$ is a strictly convex function of $u$,

$$
\eta_{u} \cdot f_{u}=F_{u},
$$

where $F$ is some function called the entropy flux.

Admissible weak solutions of (1.1) satisfy the following inequality in the distribution sense,

$$
\eta(u)_{, t}+F(u)_{, x} \leqslant 0 \quad \forall \eta \text { verifying (1.2). }
$$

In the scalar case $(m=1)$, the condition (1.3) guarantees uniqueness of the solution to (1.1) in the range of admissible solutions. For $m$ greater than 1 , the results are weaker (see, e.g., DiPerna [2]).

1.2. Numerical Schemes and Consistency. We consider finite difference approximation of (1.1) in conservation form: $h$ is the space step, $k$ the time step, $r=$ $k / h ; U^{h}(x, t)$ is the approximate solution: $U^{h}(x, t)=U_{j}^{n}$ for $(x, t) \in\left(\left(j-\frac{1}{2}\right) h\right.$, $\left.\left(j+\frac{1}{2}\right) h\right) \times[k n, k(n+1)) ; \Delta_{+}$is the spatial difference operator: $\Delta_{+} a_{j}=a_{j+1}-a_{j}$.

$$
U_{j}^{n+1}=U_{j}^{n}-r \Delta_{+}\left(g\left(U_{j-q+1}^{n}, \ldots, U_{j+p-1}^{n}\right)\right)
$$

is the general form of a $(p+q)$-point scheme in conservation form, $g$ is the numerical flux of the scheme; we note

$$
g\left(U_{j-q+1}^{n}, \ldots, U_{j+p-1}^{n}\right)=g_{j-1 / 2}^{n} .
$$

Definition 1.1. The scheme (1.4) is consistent with (1.1) if

$$
g(U, \ldots, U, \ldots, U)=f(U)
$$

and $g$ is $K$-Lipschitz,

$$
\exists K>0 ; \quad\left|g_{j-1 / 2}-f\left(U_{j}\right)\right| \leqslant K \sum_{l=-(q-2)}^{l=(p-1)}\left|\Delta_{+} U_{j+l-1}\right| .
$$

Definition 1.2. The scheme (1.4) is consistent with the entropy condition (1.3) if:

For each $\eta$, the entropy of (1.1), there exists some numerical entropy flux $\tilde{F}$ (function of $p+q$ arguments) which satisfies

(i) $\tilde{F}(U, \ldots, U, \ldots, U)=F(U)$;

(ii) $\tilde{F}$ is $K$-Lipschitz in the sense of (1.6);

(iii) $\eta\left(U_{j}^{n+1}\right)-\eta\left(U_{j}^{n}\right)-r \Delta_{+} \tilde{F}_{j-1 / 2}^{n} \leqslant 0$.

The following theorem, due to Lax (see [5]), shows the interest of these two concepts. 
THEOREM 1.1. A limit solution of a numerical scheme satisfying Definition 1.1 (consistency with the system) is a weak solution of the initial value problem (1.1). Moreover, if the numerical scheme satisfies Definition 1.2 (consistency with the entropy condition), then the limit solution is a weak admissible solution of the I.V.P. (1.1).

Remark. We call limit solution a limit in the sense of bounded $L_{\text {loc }}^{1}$ convergence (i.e., $U^{h}$ converges towards $U$ in $L_{\text {loc }}^{1}$ as $h$ goes to zero and $U^{h}$ is bounded in $L^{\infty}$ ).

\section{Numerical Schemes.}

2.1. Godunov Type Schemes. In [5], Harten, Lax and Van-Leer define a class of approximate Riemann solvers and their associated Godunov type schemes. They give two properties that allow numerical schemes to verify Definitions 1.1 and 1.2 (we refer the reader to [5] for more details).

The canonical example of Godunov type schemes is the Godunov scheme: The exact solution is taken as the Riemann solver; we can easily prove that it satisfies Definition 1.1 and it is known to be consistent with the entropy condition when the generic Riemann solution exists.

We can only prove such a result in the scalar case, in some particular cases (e.g., Euler isentropic equations) and for some particular entropy (in the last case, $L^{\infty}$ stability is also proved, see Le Roux [8]). The problems are similar for any other Godunov type scheme.

The only known example in which we can prove that a scheme satisfies the exact Definition 1.2 is the Lax-Friedrichs scheme (see Lax [7]). In [5], Harten, Lax and Van-Leer present a one-intermediate-state Riemann solver,

$$
\begin{gathered}
u\left(\frac{x}{t}, u_{L}, u_{R}\right)= \begin{cases}u_{L} & \text { if } \frac{x}{t}<a_{L}, \\
u_{L R} & \text { if } a_{L}<\frac{x}{t}<a_{R}, \\
u_{R} & \text { if } a_{R}<\frac{x}{t},\end{cases} \\
u_{L R}=\frac{a_{R} u_{R}-a_{L} u_{L}}{a_{R}-a_{L}}-\frac{f_{R}-f_{L}}{a_{R}-a_{L}} .
\end{gathered}
$$

They show consistency with the entropy condition under the following restriction:

$a_{L}$ and $a_{R}$ are lower and upper bounds, respectively, for the smallest and highest signal velocity in the Riemann problem. For $m$ greater than 1 , there is no general method for calculating such bounds (unless $\left|u_{L}-u_{R}\right|$ is small). For the particular case of Euler isentropic equations, we present in [14] an algorithm that calculates some $a_{L}$ and $a_{R}$ and hence we obtain consistency with entropy condition for the physical choice of entropy ("mechanical energy").

2.2. Scalar Case and TVD Schemes. In the scalar case, a basic tool for inducing convergence of a numerical scheme is the concept of TVD. We study it in detail and show that any 3-point TVD scheme in conservation form is at most first-order accurate. This shows that schemes with more than 3 points are required to achieve second-order accuracy.

2.2.1. 3-point TVD schemes (in conservation form). Let

$$
\operatorname{TV}(U)=\sum_{j}\left|\Delta_{+} U_{j}\right|
$$


Consider

$$
U_{j}^{n+1}=U_{j}^{n}-r \Delta_{+} g\left(U_{j-1}^{n}, U_{j}^{n}\right) .
$$

Definition 2.1. The scheme (2.1) is TVD if and only if $\operatorname{TV}\left(U^{n+1}\right) \leqslant \operatorname{TV}\left(U^{n}\right)$.

A 3-point scheme is characterized by its numerical viscosity

$$
Q_{j+1 / 2}^{n}=r \frac{f\left(U_{j}^{n}\right)-2 g\left(U_{j}^{n}, U_{j+1}^{n}\right)+f\left(U_{j+1}^{n}\right)}{\Delta_{+} U_{j}^{n}} .
$$

Hence, the scheme (2.1) can be written as

$$
U_{j}^{n+1}=U_{j}^{n}-\frac{r}{2}\left(f\left(U_{j+1}^{n}\right)-f\left(U_{j-1}^{n}\right)\right)+\frac{1}{2} \Delta_{+}\left(Q_{j-1 / 2}^{n} \Delta_{+} U_{j-1}^{n}\right) .
$$

A 3-point scheme is also characterized by its incremental form:

$$
C_{j+1 / 2}^{+, n}=r \frac{f_{j}^{n}-g_{j+1 / 2}^{n}}{\Delta_{+} U_{j}^{n}} \quad\left(g_{j+1 / 2}^{n} \equiv g\left(U_{j}^{n}, U_{j+1}^{n}\right) ; f_{j}^{n} \equiv f\left(U_{j}^{n}\right)\right),
$$

$$
\begin{gathered}
C_{j+1 / 2}^{-, n}=r \frac{f_{j+1}^{n}-g_{j+1 / 2}^{n}}{\Delta_{+} U_{j}^{n}}, \\
U_{J}^{n+1}=U_{j}^{n}+C_{j+1 / 2}^{+, n} \Delta_{+} U_{j}^{n}-C_{j-1 / 2}^{-, n} \Delta_{+} U_{j-1}^{n} .
\end{gathered}
$$

The coefficients $C_{j+1 / 2}^{ \pm}$are related to the numerical viscosity by

$$
C_{j+1 / 2}^{ \pm, n}=\frac{1}{2}\left(Q_{j+1 / 2}^{n} \mp r \frac{\Delta_{+} f_{j}^{n}}{\Delta_{+} U_{j}^{n}}\right) .
$$

We note that $C_{j+1 / 2}^{+. n}, Q_{j+1 / 2}^{n}, g_{j+1 / 2}^{n}$ are functions of two arguments: $U_{j}^{n}$ and $U_{j+1}^{n}$.

THEOREM 2.1. A 3-point TVD scheme is at most first-order accurate (in smooth regions).

Similar results can be found in Harten [3] and in Tadmor [13]; we therefore omit the proof and just recall some interesting lemmas.

LEMMA 2.1. Let a scheme be given in its incremental form (2.5). We assume that its incremental coefficients are positive. We denote by (i) and (ii) the following conditions on these coefficients:

(i) $C_{j+1 / 2}^{+}+C_{j+1 / 2}^{-} \leqslant 1 \forall j \in \mathbf{Z}$,

(ii) $C_{j+3 / 2}^{+}-C_{j+1 / 2}^{-} \leqslant 1 \forall j \in \mathbf{Z}$.

If condition (i) is satisfied, we have $\operatorname{TV}\left(U^{n+1}\right) \leqslant \operatorname{TV}\left(U^{n}\right)$. If condition (ii) is satisfied, we have $\left\|U^{n+1}\right\|_{\infty} \leqslant\left\|U^{n}\right\|_{\infty}$.

Lemma 2.2. A 3-point scheme in conservation form is completely determined by its numerical viscosity $Q$, or by its incremental coefficients $C^{+}, C^{-}$. The following conditions are equivalent:

(i) (2.1) is a 3-point TVD scheme;

(ii) $1 \geqslant Q_{j+1 / 2} \geqslant r\left|\Delta_{+} f_{j} / \Delta_{+} U_{j}\right| \forall j \in \mathbf{Z}$;

(iii) $C_{j+1 / 2}^{+}+C_{j+1 / 2}^{-} \leqslant 1, C_{j+1 / 2}^{+} \geqslant 0, C_{j+1 / 2}^{-} \geqslant 0, \forall j \in \mathbf{Z}$.

We now recall a recent result of Tadmor [12] on TVD schemes in the scalar case. 
THEOREM 2.2. A 3-point TVD scheme containing more numerical viscosity than the Godunov scheme is consistent with the entropy condition under the Courant-FriedrichsLevy (CFL) condition $1 / 2$, and it converges towards the unique entropy-satisfying solution.

\section{High-Order Schemes.}

3.1. General Algorithm and Main Theorem. We present here an algorithm that converts a 3-point first-order accurate scheme in conservation form to a 7-point more accurate scheme in conservation form.

Suppose the initial 3-point scheme satisfies Definition 1.2 (consistency with the entropy condition). Then we shall prove this algorithm to ensure that limit solutions are weak entropy-satisfying solutions of the initial value problem.

We first compute $\bar{U}_{j}^{n+1}$ from $U_{j}^{n}$ with the 3-point scheme, which can be considered as a predictor

$$
\bar{U}_{j}^{n+1}=U_{j}^{n}-r \Delta_{+} g_{j-1 / 2}^{n} .
$$

We then compute antidiffusion vectors $a_{j+1 / 2}^{n+1}=\left(a_{j+1 / 2}^{1, n+1}, \ldots, a_{j+1 / 2}^{l, n+1}, \ldots, a_{j+1 / 2}^{m, n+1}\right)$,

$$
\begin{aligned}
a_{j+1 / 2}^{n+1}= & \frac{r}{2}\left(f\left(U_{j+1}^{n}\right)-2 g_{j+1 / 2}^{n}+f\left(U_{j}^{n}\right)\right) \\
& -\frac{r^{2}}{2} D f_{j+1 / 2}^{n} \cdot\left(f\left(U_{j+1}^{n}\right)-f\left(U_{j}^{n}\right)\right) .
\end{aligned}
$$

$D f_{j+1 / 2}^{n}$ is some approximation of $D f(U)$ (the Jacobian of $\left.f(U)\right)$ near $U_{j}^{n}$ and $U_{j+1}^{n}$.

If we compute $U_{j}^{n+1}$ by

$$
U_{j}^{n+1}=\bar{U}_{j}^{n+1}-\Delta_{+} a_{j-1 / 2}^{n+1},
$$

we obtain the well-known Lax-Wendroff second-order accurate scheme which admits unstable and entropy-violating solutions. A correction of antidiffusion vectors is necessary to achieve stability and good entropy production. We thus use a correction based on the Boris and Book FCT algorithm [1] (see also Le Roux and Quesseveur [9]), $\forall l \in\{1,2, \ldots, m\}$

$$
\left\{\begin{array}{l}
\sigma^{\prime}=\operatorname{sgn} \Delta_{+} \bar{U}_{j}^{l, n} \\
\bar{a}_{j+1 / 2}^{l, n}=\sigma^{\prime} \max \left\{0, \min \left(C h^{\alpha},\left|a_{j+1 / 2}^{l, n}\right|, \frac{1}{2} \sigma^{\prime} \Delta_{+} \bar{U}_{j+1}^{l, n}, \frac{1}{2} \sigma^{l} \Delta_{+} \bar{U}_{j-1}^{l, n}\right)\right\} .
\end{array}\right.
$$

$C$ is some positive constant and $\alpha$ a chosen real number such that $0<\alpha<1$. The final solution at time step $n+1$ is computed as follows:

$$
U_{j}^{n+1}=\bar{U}_{j}^{n+1}-\Delta_{+} \bar{a}_{j-1 / 2}^{n+1} \text {. }
$$

We can briefly describe our scheme as a two-step algorithm: a prediction step (3.1) and an antidiffusion-correction step (3.2), (3.4), (3.5).

We now state our main results; proofs will be given in Subsection 3.2.

THEOREM 3.1. Let a 2-step scheme be defined by

$$
\begin{gathered}
\bar{U}_{j}^{n+1}=U_{j}^{n}-r \Delta_{+} g_{j-1 / 2}^{n}, \\
U_{j}^{n+1}=\bar{U}_{j}^{n+1}-\Delta_{+} \bar{a}_{j-1 / 2}^{n+1} .
\end{gathered}
$$


Assume that

(i) the predictor scheme (3.6) is consistent with the conservation law (1.1) and its entropy condition (in the sense of Definitions 1.1 and 1.2);

(ii) the "antidiffusion coefficients" $\bar{a}_{j-1 / 2}$ satisfy

$$
\left|\bar{a}_{j-1 / 2}\right| \leqslant e(h) \quad \forall j \in \mathbf{Z} \text { with } \lim _{h \rightarrow 0} e(h)=0 .
$$

Then, if the approximate solution $U^{h}(x, t)$ (computed by (3.6)-(3.7)) is bounded in TV and $L^{\infty}, U^{h}$ converges boundedly towards some $u$ in $L_{\mathrm{loc}}^{1}$ and $u$ is a weak entropy-satisfying solution of the I.V.P. (1.1).

Remark 3.1. In the scalar case we can take $D f_{j+1 / 2}^{n}=\Delta_{+} f\left(U_{j}^{n}\right) / \Delta_{+} U_{j}^{n}$ and

$$
a_{j+1 / 2}^{n+1}=\frac{1}{2}\left(Q_{j+1 / 2}^{n}-r^{2}\left(\frac{\Delta_{+} f\left(U_{j}^{n}\right)}{\Delta_{+} U_{j}^{n}}\right)^{2}\right) \Delta_{+} U_{j}^{n},
$$

which exhibits the numerical viscosity coefficients.

We also claim that our algorithm preserves the "TVDness" of the 3-point schemes. It converts a 3-point TVD scheme to a 7-point second-order accurate TVD scheme. This will be proved by the following theorem.

THEOREM 3.2. $m=1$ (scalar case). Suppose the predictor scheme is TVD and satisfies Definitions 1.1 and 1.2 (consistency with the system and its entropy condition). Then the approximate solution $U^{h}$, calculated by our 2-step algorithm converges towards the unique admissible solution $u$ of the IVP (1.1).

In particular, if the numerical viscosity $Q$ of the predictor scheme satisfies

$$
Q_{j+1 / 2}^{G}<Q_{j+1 / 2}<1 / 2
$$

(where $Q_{j+1 / 2}^{(i}$ is the numerical viscosity of the Godunov scheme), the hypotheses of Theorem 3.1 are satisfied and the final scheme is second-order accurate in the regions where the solution is smooth.

\subsection{Proof of Main Results.}

3.2.1. A general form of correction and its properties. To clarify the proof of Theorem 3.1, it is useful to examine some properties of a general algorithm (including (3.1), (3.2), (3.4), (3.5)).

Definition 3.1. Consider the following algorithm:

$$
U_{j}^{n+1}=\bar{U}_{j}^{n+1}-\Delta_{+} \bar{a}_{j-1 / 2}^{n+1}
$$

with $\bar{U}_{j}^{n+1}$ given by some scheme $\bar{U}_{j}^{n+1}\left(h, k, U_{j-1}^{n}, U_{j}^{n}, U_{j+1}^{n}\right)$.

(i) The algorithm is said to be a M.C. (monotone correction) if and only if

$$
\forall l \in\{1,2, \ldots, m\} \quad \bar{a}_{j+1 / 2}^{l, n}=\mu_{j+1 / 2}^{l, n} \Delta_{+} \bar{U}_{j+1}^{l, n}=\delta_{j+1 / 2}^{l, n} \Delta_{+} \bar{U}_{j-1}^{l, n}
$$

with

(3.11c) $\mu_{j+1 / 2}^{\prime, n} \geqslant 0, \quad \delta_{j+1 / 2}^{l, n} \geqslant 0, \quad \delta_{j+3 / 2}^{l, n}+\mu_{j+1 / 2}^{l, n} \leqslant 1, \quad \delta_{j+3 / 2}^{l, n}+\mu_{j-1 / 2}^{l, n} \leqslant 1$;

(ii) an M.C. correction is said to be $\mathrm{MC}_{e}$ if and only if there exists a function $e(h)$, positive, such that $\lim _{h \rightarrow 0} e(h)=0$ and

$$
\left|\bar{a}_{j+1 / 2}^{l, n}\right| \leqslant e(h)
$$


Proposition 3.1. The correction algorithm (3.2), (3.4), (3.5) is $\mathrm{MC}_{e}$ with $e(h)=$ $C h^{\alpha}$.

Proof. $\bar{a}_{j+1 / 2}^{l}$ is defined by the following identity

$$
\bar{a}_{j+1 / 2}^{\prime}=\sigma^{\prime} \max \left(0, \min \left\{C h^{\alpha},\left|a_{j+1 / 2}^{l}\right|, \frac{1}{2} \sigma^{\prime} \Delta_{+} \bar{U}_{j-1}^{\prime}, \frac{1}{2} \sigma^{\prime} \Delta_{+} \bar{U}_{j+1}^{\prime}\right) .\right.
$$

So we get

- either

$$
\begin{gathered}
\operatorname{sgn} \Delta_{+} \bar{U}_{j-1}^{\prime}=\operatorname{sgn} \Delta_{+} \bar{U}_{j}^{l}=\operatorname{sgn} \Delta_{+} \bar{U}_{j+1}^{l}=\sigma^{\prime}, \\
\bar{a}_{j+1 / 2}^{\prime}=\sigma^{\prime} \min \left\{C h^{\alpha},\left|a_{j+1 / 2}^{l}\right|, \frac{1}{2}\left|\Delta_{+} \bar{U}_{j-1}^{l}\right|, \frac{1}{2}\left|\Delta_{+} \bar{U}_{j+1}^{l}\right|\right\},
\end{gathered}
$$

thus $\left|\bar{a}_{j+1 / 2}^{l}\right| \leqslant C h^{\alpha}, \bar{a}_{j+1 / 2}^{l}=\delta \Delta_{+} \bar{U}_{j-1}^{l}=\mu \Delta_{+} \bar{U}_{j+1}^{l}$, with $0 \leqslant \mu \leqslant \frac{1}{2}$ and then $0 \leqslant \delta$ $\leqslant \frac{1}{2}$, so that inequalities $(3.11 \mathrm{~b}, \mathrm{c})$ are satisfied;

-or $\bar{a}_{j+1 / 2}^{\prime}=0$, in which case $(3.11 \mathrm{~b}, \mathrm{c})$ again holds.

We now present the main property of M.C. schemes.

Proposition 3.2. An M.C. correction does not increase the TV and $L^{\infty}$ norms:

$$
\begin{aligned}
\left\|U^{l, n+1}\right\|_{\infty} & \leqslant\left\|\bar{U}^{l, n+1}\right\|_{\infty}, \\
\operatorname{TV}\left(U^{l, n+1}\right) & \leqslant \operatorname{TV}\left(\bar{U}^{l, n+1}\right) .
\end{aligned}
$$

Proof of Proposition 3.2. Substituting for $a_{j \pm 1 / 2}$ from (3.11b) in (3.11a), we obtain the following incremental form for $U_{j}^{l, n+1}$ :

$$
U_{j}^{l, n+1}=\bar{U}_{j}^{l, n+1}-\delta_{j+1 / 2}^{l, n+1} \Delta_{+} \bar{U}_{j-1}^{l, n+1}+\mu_{j-1 / 2}^{l, n+1} \Delta_{+} \bar{U}_{j}^{l, n+1} .
$$

Taking relation (3.11c) into account, we note that the incremental coefficients are positive and satisfy hypotheses (i) and (ii) of Lemma 2.1 with

$$
C_{j-1 / 2}^{-}=\delta_{j+1 / 2}^{l, n+1}, \quad C_{j+1 / 2}^{+}=\mu_{j-1 / 2}^{l, n+1} .
$$

The results now follow immediately.

\subsubsection{Proof of Theorem 3.1.}

- Convergence towards a weak solution. This part of the proof is straightforward, since the scheme is in conservation form, and is therefore omitted.

- Entropy condition. This is the crucial part of the proof.

We use the approximate entropy inequality associated with the scheme (3.1),

$$
\eta\left(\bar{U}_{j}^{n+1}\right)-\eta\left(U_{j}^{n}\right)+r \Delta_{+} F_{j-1 / 2}^{n} \leqslant 0 .
$$

We denote by $F^{h}(x, t)$ the function

$$
F^{h}(x, t)=F_{j-1 / 2}^{n} \quad \text { for }(x, t) \in((j-1) h, j h) \times[k n, k(n+1))
$$

and by $\phi$ some positive test function in $C_{0}^{\infty}$,

$$
\begin{array}{ll}
\phi_{. t}^{h}(x, t)=\frac{\phi_{j}^{n+1}-\phi_{j}^{n}}{k} \quad \text { for }(x, t) \in\left(\left(j-\frac{1}{2}\right) h,\left(j+\frac{1}{2}\right) h\right) \times[k n, k(n+1)), \\
\phi_{. x}^{h}(x, t)=\frac{\phi_{j}^{n}-\phi_{j-1}^{n}}{h} \quad \text { for }(x, t) \in((j-1) h, j h] \times[k n, k(n+1)) .
\end{array}
$$


We multiply (3.17) by $h \phi_{j}^{n}$ and sum over $n$ and $j$,

$$
\begin{gathered}
\sum_{n, j} h\left(\eta\left(U_{j}^{n+1}\right)-\eta\left(U_{j}^{n}\right)\right) \phi_{j}^{n}+\sum_{n, j} k \Delta_{+}\left(F_{j-1 / 2}^{n}\right) \phi_{j}^{n} \\
\leqslant \sum_{n, j} h\left(\eta\left(U_{j}^{n+1}\right)-\eta\left(\bar{U}_{j}^{n+1}\right)\right) \phi_{j}^{n} .
\end{gathered}
$$

In the sequel we denote by RS (resp. LS) the right (resp. left) side of inequality (3.18). Integrating LS by parts with respect to each increment, the left-hand side of (3.18) becomes

$$
-\sum_{n, j} h k \eta\left(U_{j}^{n+1}\right) \frac{\phi_{j}^{n+1}-\phi_{j}^{n}}{k}-\sum_{n, j} h k F_{j+1 / 2}^{n} \frac{\phi_{j+1}^{n}-\phi_{j}^{n}}{h},
$$

which turns out to be equal to

$$
-\iint_{\mathbf{R} \times \mathbf{R}^{+}} d x d t\left\{\eta\left(U^{h}(x, t)\right) \phi_{, t}^{h}(x, t)+F^{h}(x, t) \phi_{, x}^{h}(x, t)\right\} .
$$

The convergence of LS towards the quantity

$$
-\iint d x d t\left\{\eta(u(x, t)) \phi_{, t}(x, t)+F(x, t) \phi_{, x}(x, t)\right\}
$$

follows immediately from the Lebesgue dominated convergence theorem and the $K$-Lipschitz continuity of $F^{h}$ (see Definition 1.2).

It remains to prove that $\overline{\lim }_{h \rightarrow 0} \mathrm{RS} \leqslant 0$. We shall use a consequence of the convexity of $\eta$ :

If $\eta$ is a $\mathscr{C}^{2}$ convex function in $\mathbf{R}^{m}$, then

$$
\forall(X, Y) \in\left(\mathbf{R}^{m}\right)^{2}, \quad \eta(X)-\eta(Y) \leqslant \eta^{\prime}(X) \cdot(X-Y) .
$$

Thus,

$$
\mathrm{RS} \leqslant \sum_{n, j} \phi_{j}^{n} h \eta^{\prime}\left(U_{j}^{n+1}\right) \cdot\left(U_{j}^{n+1}-\bar{U}_{j}^{n+1}\right) \equiv \mathrm{RS}_{1}
$$

By (3.11a),

$$
\mathrm{RS}_{1}=-\sum_{n, j} h \phi_{j}^{n} \eta^{\prime}\left(U_{j}^{n+1}\right) \cdot \Delta_{+} \bar{a}_{j+1 / 2}^{n+1}
$$

Integrating by parts, we obtain

$$
\begin{aligned}
\mathrm{RS}_{1}= & \sum_{n, j} h \Delta_{+}\left(\phi_{j}^{n} \eta^{\prime}\left(U_{j}^{n+1}\right)\right) \cdot \bar{a}_{j-1 / 2}^{n+1}=\sum_{n, j} h \phi_{j+1}^{n}\left(\Delta_{+} \eta^{\prime}\left(U_{j}^{n+1}\right)\right) \cdot \bar{a}_{j-1 / 2}^{n+1} \\
& +\sum_{n, j} h\left(\Delta_{+} \phi_{j}^{n}\right) \eta^{\prime}\left(U_{j}^{n+1}\right) \cdot \bar{a}_{j-1 / 2}^{n+1} .
\end{aligned}
$$

We denote by $\mathrm{RS}_{2}$ and $\mathrm{RS}_{3}$ respectively the first and second sum in the right-hand side of the previous equality, thus $\mathrm{RS}_{1}=\mathrm{RS}_{2}+\mathrm{RS}_{3}$.

We examine first $\mathbf{R S}_{2}$. Since $\phi$ has compact support, we can assume that $\phi(x, t) \equiv 0$ for $|t|>T$. Furthermore, since $U^{h}$ is bounded in $L^{\infty}$, we have

$$
\left|\Delta_{+} \eta^{\prime}\left(U_{j}^{n+1}\right)\right| \leqslant\left\|\eta^{\prime \prime}\left(U^{n}(\cdot,(n+1) k)\right)\right\|_{\infty}\left|U_{j+1}^{n+1}-U_{j}^{n+1}\right| \leqslant K_{1}\left|\Delta_{+} U_{j}^{n+1}\right| .
$$

We can thus write

$$
\mathrm{RS}_{2} \leqslant K_{1} e(h) \sum_{n, j} h \phi_{j+1}^{n}\left|\Delta_{+} U_{j}^{n+1}\right| \leqslant \frac{h}{k} K_{1} e(h)\|\phi\|_{\infty} \sum_{n k \leqslant T} k \operatorname{TV}\left(U^{n+1}\right) .
$$


Since $U^{h}(\cdot, t)$ is bounded in TV, and $h / k$ is bounded, there exists a constant $K_{2}$ such that

$$
\mathrm{RS}_{2} \leqslant K_{2}(\phi, T, h / k) e(h) .
$$

We next analyze $\mathrm{RS}_{3}$. We have

$$
\mathrm{RS}_{3} \leqslant \frac{h}{k} e(h) \sum_{n, j} k h \frac{\left|\Delta_{+} \phi_{j}\right|}{h}\left|\eta^{\prime}\left(U_{j}^{n+1}\right)\right| \equiv \mathrm{RS}_{4} .
$$

By introducing $\phi_{, x}^{h}$ and $\eta^{\prime}\left(U^{h}\right)$, the step functions associated with $\Delta_{+} \phi_{j}^{n} / h$ and $\eta^{\prime}\left(U_{j}^{n+1}\right)$, respectively, we get

$$
\mathrm{RS}_{4}=\frac{h}{k} e(h) \iint d x d t\left|\phi_{, x}^{h}(x, t) \| \eta^{\prime}\left(U^{h}(x, t)\right)\right| .
$$

By the Lebesgue dominated convergence theorem there exists a positive constant $C$ such that

$$
\mathrm{RS}_{4} \leqslant \mathrm{Ce}(h)
$$

Combining relations (3.20) and (3.21), we obtain $\lim _{h \rightarrow 0} \mathrm{RS}=0$.

3.2.3. Proof of Theorem 3.2. The first part (convergence) is a straightforward corollary of Theorem 3.1. Using BV estimates together with $L^{\infty}$ estimates (the initial condition is supposed to have a compact support), we obtain $L^{1}$ estimates. Compactness results of $L_{\text {loc }}^{\prime} \cap \mathrm{BV}$ in $L_{\text {loc }}^{\prime}$ permit us to construct a subsequence $U^{h}$ converging towards the unique admissible solution $u$.

- Second-order accuracy. We shall discuss two complementary points of view:

- Characterizing the "smooth regions" in which our scheme reduces to the Lax-Wendroff scheme.

- Proving directly second-order accuracy by the use of standard Taylor expansion methods.

We first have to characterize the "smooth regions". We use boundedness of derivatives and we limit ourselves to regions far away from critical points of $u$. We shall take (R.C.) as a regularity criterion near $x=j_{0} h$ at time $n k$ : .

$$
\left\{\begin{array}{lll}
\text { (i) } & \Delta_{+} U_{j}^{n} \text { retains a constant sign for } j \in\left[j_{0}-p, j_{0}+p\right], p \in \mathbf{N}, \\
\text { (ii) } & \left|\Delta_{+} U_{j}^{n} / h\right| \leqslant C_{R} \quad \forall j \in\left[j_{0}-p+1, j_{0}+p-1\right] ; & p>2 ; \\
\text { (iii) } & \min \left(\frac{\left|\Delta_{+} U_{j}^{n}\right|}{\left|\Delta_{+} U_{j-1}^{n}\right|}, \frac{\left|\Delta_{+} U_{j-1}^{n}\right|}{\left|\Delta_{+} U_{j}^{n}\right|}\right) \geqslant \frac{5}{8} \quad \forall j \in\left[j_{0}-p+2, j_{0}+p-2\right] .
\end{array}\right.
$$

We make the following comments about (R.C.): (i) expresses the monotonicity, (ii) the boundedness of the first spatial derivative; criterion (iii) is similar to the boundedness of the second spatial derivative. From (3.9) we have

$$
a_{j+1 / 2}^{n+1}=\frac{1}{2}\left(Q_{j+1 / 2}^{n}-r^{2}\left|\frac{\Delta_{+} f_{j}^{n}}{\Delta_{+} U_{j}}\right|^{2}\right) \Delta_{+} U_{j}^{n} .
$$

We shall take

$$
\bar{a}_{j+1 / 2}^{n+1}=\sigma \max \left\{0, \min \left\{\frac{1}{4} C_{R} h^{\alpha},\left|a_{j+1 / 2}^{n+1}\right|, \frac{1}{2} \sigma \Delta_{+} \bar{U}_{j+1}^{n+1}, \frac{1}{2} \sigma \Delta_{+} \bar{U}_{j+1}^{n+1}\right\}\right\}
$$


and prove that $\bar{a}_{j+1 / 2}^{n+1}=a_{j+1 / 2}^{n+1}$; therefore, the scheme is second-order accurate near $x_{j}$, since it has the same numerical flux as the Lax-Wendroff scheme.

For simplicity we assume $\Delta_{+} U_{j}^{n}>0$; then $a_{j+1 / 2}^{n+1}$ is positive, and from (3.10) we have

$$
\left|a_{j+1 / 2}^{n+1}\right| \leqslant \frac{1}{4} \Delta_{+} U_{j}^{n} \leqslant \frac{1}{4} C_{R} h .
$$

By using the increment form of the predictor scheme, we get

$$
\begin{aligned}
\Delta_{+} \bar{U}_{j+1}^{n+1}= & \left(1-Q_{j+3 / 2}^{n}\right) \Delta_{+} U_{j+1}^{n}+\frac{1}{2}\left(Q_{j+5 / 2}^{n}-r\left(\frac{\Delta_{+} f_{j+2}^{n}}{\Delta_{+} U_{j+2}^{n}}\right)\right) \Delta_{+} U_{j+2}^{n} \\
& +\frac{1}{2}\left(Q_{j+1 / 2}^{n}+r \frac{\Delta_{+} f_{j}^{n}}{\Delta_{+} U_{j}^{n}}\right) \Delta_{+} U_{j}^{n} .
\end{aligned}
$$

We set $\chi=\Delta_{+} f_{j}^{n} / \Delta_{+} U_{j}^{n}$.

By using (R.C.) (iii) and (3.10), we are led to

Then

$$
\Delta_{+} \bar{U}_{j+1}^{n+1} \geqslant\left(\left(\frac{5}{16}+\frac{1}{2}\left(Q_{j+1 / 2}+r \chi\right)\right) \Delta_{+} U_{j}^{n}\right) \text {. }
$$

$$
\begin{aligned}
\frac{2}{\Delta_{+} U_{j}^{n}}\left(a_{j+1 / 2}^{n+1}-\frac{1}{2} \Delta_{+} \bar{U}_{j+1}^{n+1}\right) & \leqslant \frac{1}{2} Q_{j+1 / 2}^{n}-\frac{5}{16}-\frac{1}{2} r \chi-r^{2} \chi^{2} \\
& \leqslant-\frac{1}{2} r \chi-r^{2} \chi^{2}-\frac{1}{16}=-\left(r \chi+\frac{1}{4}\right)^{2} \leqslant 0,
\end{aligned}
$$

and therefore $\left|a_{j+1 / 2}^{n+1}\right| \leqslant \frac{1}{2} \Delta_{+} \bar{U}_{j+1}^{n+1}$. Similarly, we obtain $\left|a_{j+1 / 2}^{n+1}\right| \leqslant \frac{1}{2} \Delta_{+} \bar{U}_{j-1}^{n+1}$, hence $\bar{a}_{j+1 / 2}^{n+1}=a_{j-1 / 2}^{n+1}$.

- A direct proof of second-order accuracy is possible. A sufficient condition is that

$$
g_{j+1 / 2}^{n}+r \bar{a}_{j+1 / 2}^{n+1}=f_{j+1 / 2}^{n}+r a_{j+1 / 2}^{n+1}+O\left(h^{2}\right)
$$

$\left(g_{j+1 / 2}^{n}+r a_{j+1 / 2}^{n+1}\right.$ is the numerical flux of the Lax-Wendroff scheme). We shall use an equivalent condition,

$$
\bar{a}_{j+1 / 2}^{n+1}=a_{j+1 / 2}^{n+1}+O\left(h^{2}\right) .
$$

In a smooth region away from critical points of $u$, the following estimates are valid:

$$
\begin{gathered}
Q_{j+5 / 2}^{n}=Q_{j+1 / 2}^{n}+O_{1}(h), \quad Q_{j+3 / 2}^{n}=Q_{j+1 / 2}^{n}+O_{2}(h), \quad \Delta_{+} U_{j}^{n}=O_{3}(h), \\
\frac{\Delta_{+} f_{j+2}^{n}}{\Delta_{+} U_{j+2}^{n}}=\chi+O_{4}(h), \quad \Delta_{+} U_{j+1}^{n}=\Delta_{+} U_{j}^{n}\left(1+O_{5}(h)\right), \\
\Delta_{+} U_{j-1}^{n}=\Delta_{+} U_{j}^{n}\left(1+O_{6}(h)\right) .
\end{gathered}
$$

It follows that

$$
\text { (3.22) } \Delta_{+} \bar{U}_{j+1}^{n+1}=\Delta_{+} U_{j}^{n}\left(1+O_{7}(h)\right), \quad \Delta_{+} \bar{U}_{j-1}^{n+1}=\Delta_{+} U_{j}^{n}\left(1+O_{8}(h)\right) \text {. }
$$

If $\bar{a}_{j+1 / 2} \neq a_{j+1 / 2}$, either $\bar{a}_{j+1 / 2}=\frac{1}{2} \Delta_{+} \bar{U}_{j-1}$, or $\bar{a}_{j+1 / 2}=\frac{1}{2} \Delta_{+} \bar{U}_{j+1}$. Suppose first that $\bar{a}_{j+1 / 2}=\frac{1}{2} \Delta_{+} \bar{U}_{j-1}$ (it follows that $\sigma\left(a_{j+1 / 2}-\frac{1}{2} \Delta_{+} \bar{U}_{j-1}\right) \geqslant 0$; then

$$
\begin{aligned}
\sigma\left(a_{j+1 / 2}-\bar{a}_{j+1 / 2}\right) & =\frac{\left|\Delta_{+} U_{j}\right|}{2}\left(Q_{j+1 / 2}-r^{2} \chi^{2}-1+O_{8}(h)\right) \\
& \leqslant \frac{1}{2} O_{3}(h)\left(-\frac{1}{2}-r^{2} \chi^{2}+O_{8}(h)\right)
\end{aligned}
$$


which contradicts $\sigma\left(a_{j+1 / 2}-\frac{1}{2} \Delta_{+} \bar{U}_{j-1}\right) \geqslant 0$, unless

$$
Q_{j+1 / 2}-r^{2} \chi^{2}-1=O_{9}(h) .
$$

Therefore, $a_{j+1 / 2}-\bar{a}_{j+1 / 2}=O\left(h^{2}\right)$. The proof is similar if $\bar{a}_{j+1 / 2}=\frac{1}{2} \Delta_{+} \bar{U}_{j+1}$. The case $\bar{a}_{j+1 / 2}=0$ never occurs if $a_{j+1 / 2} \neq \bar{a}_{j+1 / 2}$, since, away from critical points of $u, \operatorname{sgn} \Delta_{+} \bar{U}_{j-1}=\operatorname{sgn} \Delta_{+} \bar{U}_{j+1}$ by (3.22).

3.3. Some Remarks About Second-Order Accuracy. In [3] Harten presents his second-order accurate TVD schemes as first-order TVD schemes applied to a modified equation. We shall use similar heuristic arguments to describe our algorithm.

The 3-point predictor TVD scheme is

$$
\bar{U}_{j}^{n+1}=U_{j}^{n}-r \Delta_{+} g_{j-1 / 2}^{n} \text {. }
$$

Denote

$$
\beta(u, r)=\frac{1}{2 r^{2}}\left(\frac{\partial g}{\partial u_{1}}-\frac{\partial g}{\partial u_{2}}\right)-\frac{1}{2} f^{\prime}(u)^{2} .
$$

Consider the following problem (P.1):

$$
\left\{\begin{array}{l}
\text { Find } u \text { at } t=(n+1) k \text { such that } \\
u_{, t}+f(u)_{. x}=\Delta t\left(\beta(u, r) u_{, x}\right)_{, x}, \\
u(x, n k)=U^{n} .
\end{array}\right.
$$

We can say that $\bar{U}^{n+1}$ is a second-order accurate TVD approximation to (P.1) (see, e.g., [6]).

$U^{n+1}$ is defined by $U_{j}^{n+1}=\bar{U}_{j}^{n+1}-\Delta_{+} \bar{a}_{j-1 / 2}^{n+1}$. Consider the problem (P.2):

$$
\left\{\begin{array}{l}
\text { Find } u \text { at } t=(n+1) k \text { such that } \\
u_{, t}=-\Delta t\left(\beta(u, r) u_{, x}\right)_{. x}, \\
u(x, n k)=\bar{U}^{n+1} .
\end{array}\right.
$$

We can say that $U^{n+1}$ is a second-order accurate TVD approximation to (P.2). So $U^{n+1}$ is a second-order accurate TVD approximation to (P.1), (P.2), and (P.1), (P.2) is a 2-step formulation for:

$$
\left\{\begin{array}{l}
\text { Find } u \text { at } t=(n+1) k \text { such that } \\
u_{, t}+f(u)_{. x}=0 \\
u(x, n k)=U^{n} .
\end{array}\right.
$$

4. Entropy Condition for Harten High-Order Schemes. In this section we extend the ideas developed in the previous parts to Harten's high-order schemes [3]. In the scalar case, we slightly modify them, keeping their "TVDness" and their second-order accuracy in smooth regions. We then prove that the resulting schemes converge towards the unique entropy-satisfying solution (under reasonable conditions).

4.1. A Modified Algorithm. Let us consider a 3-point TVD scheme with the following numerical viscosity:

$$
Q_{j+1 / 2}^{n}=Q\left(\nu_{j+1 / 2}^{n}\right)
$$


in which

$$
\nu_{j+1 / 2}^{n}= \begin{cases}r \frac{\Delta_{+} f\left(U_{j}^{n}\right)}{\Delta_{+} U_{j}^{n}} & \text { if } \Delta_{+} U_{j}^{n} \neq 0, \\ r f^{\prime}\left(U_{j}^{n}\right) & \text { if } \Delta_{+} U_{j}^{n}=0 .\end{cases}
$$

$Q$ is some Lipschitz function of $x$ which satisfies

$$
|x| \leqslant|Q(x)| \leqslant 1 \text { for } 0 \leqslant|x| \leqslant \mu<1 .
$$

From Lemma 2.2, the resulting scheme is TVD under the following CFL restriction:

$$
\left|\nu_{j+1 / 2}\right| \leqslant \mu \text {. }
$$

We now construct the corresponding 5-point scheme by applying the 3-point scheme to a modified flux $f^{M}$,

$$
f_{j}^{M}=f\left(U_{j}\right)+\frac{1}{r} a_{j}, \quad a_{j}=a\left(U_{j-1}, U_{j}, U_{j+1}\right) .
$$

Then

$$
\nu_{j+1 / 2}^{M}=\nu_{j+1 / 2}+\gamma_{j+1 / 2}, \quad \nu_{j+1 / 2}=\frac{\Delta_{+} a_{j}}{\Delta_{+} U_{j}} .
$$

The numerical flux of the 5-point scheme is $\bar{f}_{j+1 / 2}^{M}$,

$$
\bar{f}_{j+1 / 2}^{M}=\frac{1}{2}\left[f\left(U_{j}\right)+f\left(U_{j+1}\right)\right]+\frac{1}{2 r}\left[a_{j}+a_{j+1}-Q\left(v_{j+1 / 2}^{M}\right) \Delta_{+} U_{j}\right] .
$$

The coefficient $a_{j}$ is given by the following algorithm

$$
\left\{\begin{array}{l}
a_{j}=\sigma \max \left[0, \min \left(\left|\tilde{a}_{j+1 / 2}\right|, \tilde{a}_{j-1 / 2} \sigma, C h^{\alpha}\right)\right], \\
\tilde{a}_{j+1 / 2}=\frac{1}{2}\left[Q\left(\nu_{j+1 / 2}\right)-\left(\nu_{j+1 / 2}\right)^{2}\right] \Delta_{+} U_{j}, \\
\sigma=\operatorname{sgn}\left(\tilde{a}_{j+1 / 2}\right) .
\end{array}\right.
$$

$C$ is a positive constant, $\alpha$ a fixed real number such that $0<\alpha<1$.

LEMMA 4.1. The 5-point scheme (4.5) is TVD under the same CFL condition as the 3-point original scheme,

$$
\max _{j}\left|\nu_{j+1 / 2}\right| \leqslant \mu .
$$

It is second-order accurate in smooth regions.

Proof of Lemma 4.1. Compared to Harten's scheme, we have simply added the term $C h^{\alpha}$ in (4.5d). The new scheme is obviously as accurate as Harten's scheme, since in smooth regions $\tilde{a}_{j+1 / 2}$ is $O(h)$ (first spatial derivative of $u$ is bounded), thus $C h^{\alpha}$ is greater than $\left|\tilde{a}_{j+1 / 2}\right|$ and $\left|a_{j-1 / 2}\right|$, and therefore $a_{j}$ is the same as $g_{j}$ for the original Harten's scheme; therefore, the scheme is second-order accurate. It remains to prove that the scheme is TVD. Since it consists of the 3-point scheme applied to a modified flux, it is obviously TVD under the following CFL condition:

$$
\max _{j}\left|\nu_{j+1 / 2}^{M}\right| \leqslant 1 \text {. }
$$

Suppose $\left|\nu_{j+1 / 2}\right| \leqslant \mu$; then from (4.5d).

$$
\begin{aligned}
\left|\nu_{j+1 / 2}^{M}\right| & =\left|\nu_{j+1 / 2}+\gamma_{j+1 / 2}\right| \leqslant\left|\nu_{j+1 / 2}\right|+\frac{1}{2}\left|Q\left(\nu_{j+1 / 2}\right)-\left(\nu_{j+1 / 2}\right)^{2}\right| \\
& \leqslant\left|\nu_{j+1 / 2}\right|+\frac{1}{2}\left|1-\left(\nu_{j+1 / 2}\right)^{2}\right| \leqslant 1 .
\end{aligned}
$$


Let us consider now a simplified form of (4.5),

$$
Q\left(\nu_{j+1 / 2}^{M}\right)=Q\left(\nu_{j+1 / 2}\right)+\left|\gamma_{j+1 / 2}\right|
$$

We get as in [3] the following scheme:

$$
\begin{gathered}
U_{j}^{n+1}=U_{j}^{n}-r \Delta_{+} \bar{f}_{j-1 / 2}^{M}, \\
\bar{f}_{j+1 / 2}^{M}=\bar{f}_{j+1 / 2}+\frac{1}{r} a_{j+1 / 2} \\
\left(\bar{f}_{j+1 / 2} \text { is the numerical flux of the 3-point scheme }\right), \\
a_{j+1 / 2}=\sigma \max \left\{0, \min \left[C h^{\alpha},\left|\tilde{a}_{j+1 / 2}\right|, \sigma \tilde{a}_{j-1 / 2}, \sigma \tilde{a}_{j+1 / 2}\right]\right\} .
\end{gathered}
$$

This scheme has some resemblance with the schemes developed in Section 3. This particular form enables us to apply this construction to any 3-point TVD scheme (the numerical viscosity $Q_{j+1 / 2}$ is not necessarily a function of $\nu_{j+1 / 2}$ ). The resulting scheme has the same properties as the previous scheme (i.e., it is TVD and second-order accurate).

\subsection{Entropy Condition for the Modified Scheme.}

THEOREM 4.1. Suppose the 3-point TVD scheme satisfies Definition 1.2 (i.e., consistency with the entropy condition). Then the limit solutions of the 5-point schemes (4.5) and (4.7) are entropy-satisfying solutions of the initial value problem.

Proof. We shall use Theorem 3.2.

The scheme (4.7) has the following form: Let

$$
\bar{U}_{j}^{n+1}=U_{j}^{n}-r \Delta_{+} \bar{f}_{j-1 / 2}^{n} \text {. }
$$

Then

$$
U_{j}^{n+1}=\bar{U}_{j}^{n+1}-\Delta_{+} a_{j-1 / 2}^{n+1},
$$

and from (4.7c), $\left|a_{j+1 / 2}\right| \leqslant C h^{\alpha}$.

We get the result by a direct application of Theorem 3.2.

The scheme (4.5) also has a similar form: From (4.5c),

$$
\begin{aligned}
\bar{f}_{j+1 / 2}^{M} & =\frac{1}{2}\left[f\left(U_{j}\right)+f\left(U_{j+1}\right)\right]+\frac{1}{2 r}\left[a_{j}+a_{j+1}-Q\left(v_{j+1 / 2}^{M}\right) \Delta_{+} U_{j}\right] \\
& =\bar{f}_{j+1 / 2}+\frac{1}{2 r}\left\{a_{j}+a_{j+1}+\left[Q\left(\nu_{j+1 / 2}\right)-Q\left(\nu_{j+1 / 2}^{M}\right)\right] \Delta_{+} U_{j}\right\} .
\end{aligned}
$$

$\bar{f}_{j+1 / 2}$ is the numerical flux of the 3 -point scheme.

Let

$$
a_{j+1 / 2}=\frac{1}{2}\left\{a_{j}+a_{j+1}+\left[Q\left(\nu_{j+1 / 2}\right)-Q\left(\nu_{j+1 / 2}^{M}\right)\right] \Delta_{+} U_{j}\right\} ;
$$

then we can write the scheme as (4.8), (4.9).

From (4.10) and (4.5d),

$$
\left|a_{j+1 / 2}\right| \leqslant \frac{1}{2}\left\{C h^{\alpha}+C h^{\alpha}+\left|Q\left(\nu_{j+1 / 2}\right)-Q\left(\nu_{j+1 / 2}^{M}\right)\right| \Delta_{+} U_{j}\right\},
$$

$\left|Q\left(\nu_{j+1 / 2}\right)-Q\left(\nu_{j+1 / 2}^{M}\right)\right| \leqslant K\left|\gamma_{j+1 / 2}\right|$ since $Q$ is $K$-Lipschitz, therefore

$$
\begin{aligned}
\left|a_{j+1 / 2}\right| & \leqslant C h^{\alpha}+K\left|\gamma_{j+1 / 2}\right| \Delta_{+} U_{j} \leqslant C h^{\alpha}+K\left|a_{j+1}-a_{j}\right| \\
& \leqslant(C+2 K C) h^{\alpha} .
\end{aligned}
$$

A direct application of Theorem 3.2 gives the result. 
We shall now use the results of Tadmor [12] to make the results of Theorem 4.1 more precise.

THEOREM 4.2. Suppose the numerical viscosity $Q(x)$ satisfies

$$
Q(x)=\left\{\begin{array}{ll}
\frac{x^{2}}{4 \varepsilon}+\varepsilon & \text { for }|x|<2 \varepsilon, \\
|x| & \text { for }|x| \leqslant 2 \varepsilon,
\end{array} \quad 0<\varepsilon \leqslant \frac{1}{2} .\right.
$$

Then the scheme (4.5) converges towards the unique entropy-satisfying solution under a sufficiently small CFL condition.

Suppose the numerical viscosity of the 3-point scheme satisfies

$$
\left|\nu_{j+1 / 2}\right| \leqslant Q_{j+1 / 2}^{G} \leqslant Q_{j+1 / 2} \leqslant \frac{1}{2},
$$

where $Q_{j+1 / 2}^{G}$ is the numerical viscosity of the Godunov scheme. Then the resulting 5-point scheme converges towards the unique entropy-satisfying solution under the CFL condition

$$
\max _{j}\left|\nu_{j+1 / 2}\right| \leqslant \frac{1}{2}
$$

Moreover, if the 3-point scheme is the Godunov scheme, the result holds with

$$
\max _{j}\left|\nu_{j+1 / 2}\right| \leqslant 1
$$

Proof. Part A. From Corollary 5.1 of Tadmor [12], the 3-point scheme satisfies an approximate entropy inequality for sufficiently small CFL number. The result then follows immediately.

Part B. Here again, we make a direct application of Theorem 5.1 of Tadmor: For the Godunov scheme we can use $\mathrm{CFL}=1$, since we have an approximate entropy inequality under the same CFL restriction.

5. Numerical Experiments. We compute solutions to the Euler 1-dimensional isentropic equations of compressible gas dynamics (with $p(\rho)=k \rho^{2}$ ), using the schemes developed in the previous sections.

We first solve these equations with the following initial condition (a Riemann problem):

$$
u(x, 0)= \begin{cases}\left(\frac{37}{24}+\frac{5}{2 \sqrt{3}}, 0\right) & \text { if } x<0 \\ \left(\frac{5}{24}, 0\right) & \text { if } x>0\end{cases}
$$

The exact solution consists of a 1-rarefaction wave (containing a sonic point) and a shock wave of speed $\sigma=\frac{5}{2}$, separated by a constant state $U_{*}=\left(\frac{25}{24}, \frac{25}{12}\right)$. (See Figure 1 for a view in the $(x, t)$-plane.

We present results at time $T=4.8$, obtained respectively with

1 - the Lax-Friedrichs modified scheme (with $Q=\frac{1}{2}$ ) - (Figure 2).

2 - the Godunov scheme - (Figure 3).

3 - the L.F. modified scheme, plus the correction algorithm (3.4), (3.5)-(Figure 4).

4 - the Godunov scheme plus a M.C. correction algorithm - (Figure 5).

5 - the Godunov scheme plus the correction algorithm (3.4), (3.5)-(Figure 6). 


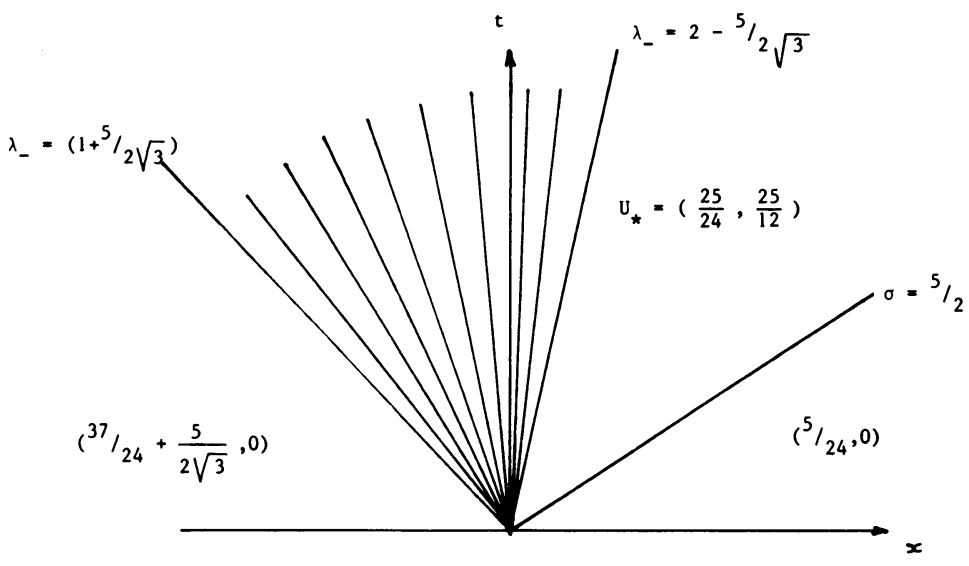

FIGURE 1

Exact solution in the $(x, t)$-plane.

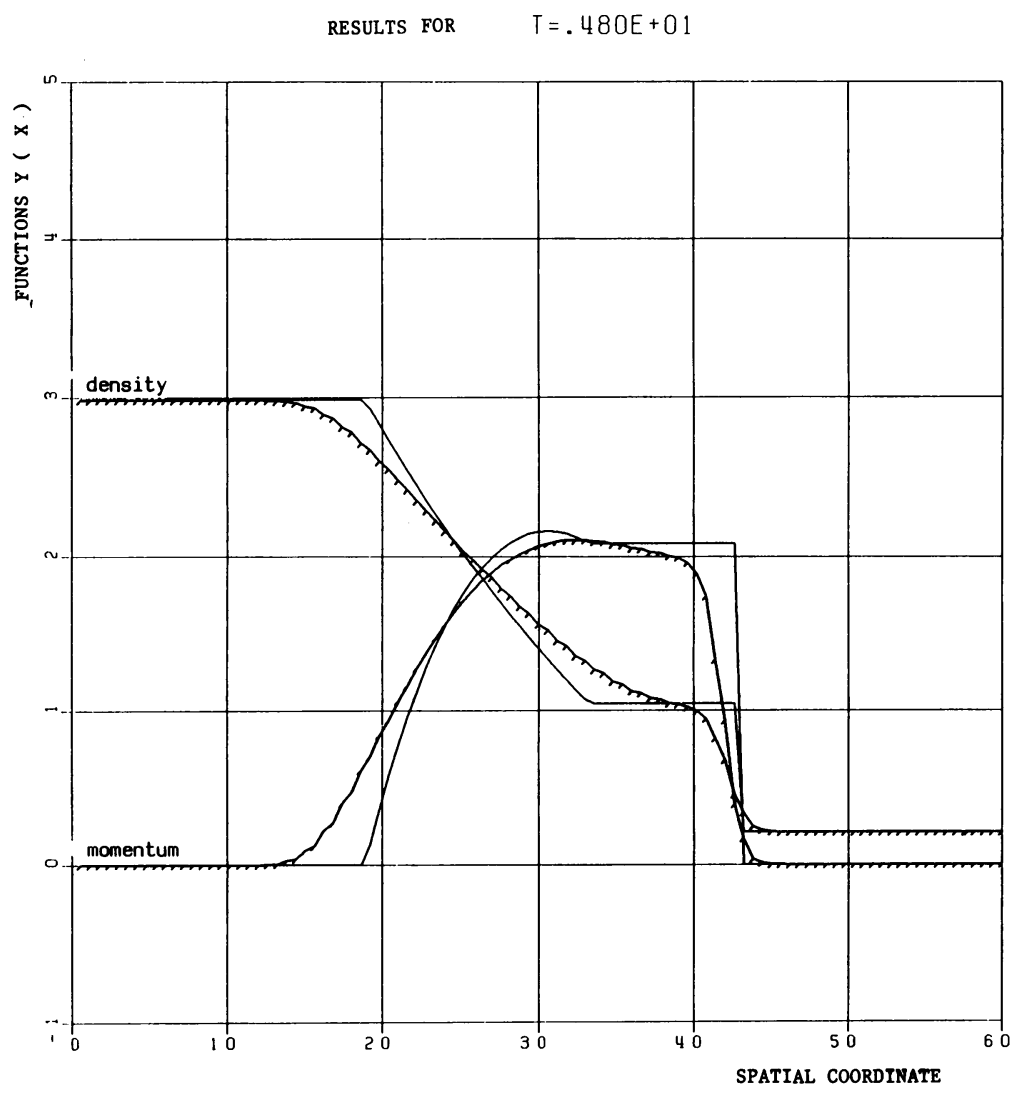

FIGURE 2

Lax-Friedrichs modified scheme

DT $=.08 \quad$ Current number $=0.5$

/ exact solution ficomputed solution 


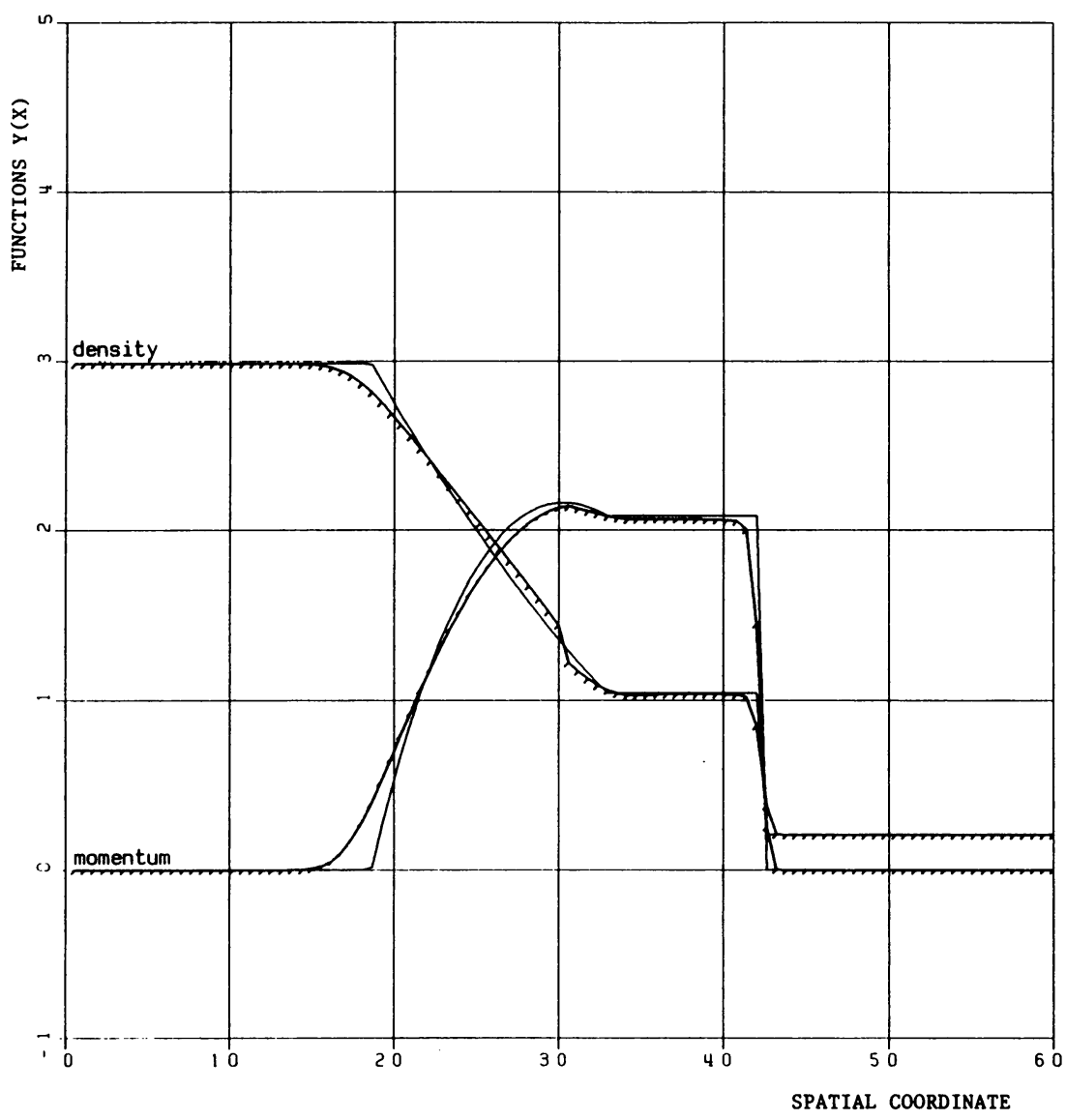

Figure 3

Godunov scheme

$D T=.16 \quad$ Current number $=1$

/exact solution computed solution

In practice, for higher-order schemes, we always omit the correction term $C h^{\alpha}$. It is only efficient in shock regions, where we already have $\bar{a}_{j+1 / 2}=0$.

In case 4, we use the following M.C. algorithm, which is more restrictive than the simplest component-by-component ones ((3.4)-(3.5)):

$a_{j+1 / 2}$ is given by (3.2) with $D f_{j+1 / 2}^{n}=D f\left(u_{E}\left(u_{j-1}, u_{j}\right)\right)\left(u_{E}\right.$ is the stationary value of the Riemann problem used in the Godunov scheme).

- As in (3.4), we define for $l=1,2$,

(i) $\left\{\begin{array}{l}\sigma^{\prime}=\operatorname{sgn} \Delta_{+} \bar{U}_{j}^{l, n}, \\ \tilde{a}_{j+1 / 2}^{\prime, n}=\sigma^{\prime} \max \left\{0, \min \left(C h^{\alpha},\left|a_{j+1 / 2}^{l, n}\right|, \frac{1}{2} \sigma^{\prime} \Delta_{+} \bar{U}_{j+1}^{l, n}, \frac{1}{2} \sigma^{\prime} \Delta_{+} \bar{U}_{j-1}^{l, n}\right)\right\},\end{array}\right.$

(ii) $\begin{cases}\bar{a}_{j+1 / 2}^{l, n}=0, & \text { if } \tilde{a}_{j+1 / 2}^{1} \cdot \tilde{a}_{j+1 / 2}^{2}=0, \\ \bar{a}_{j+1 / 2}^{l, n}=\tilde{a}_{j+1 / 2}^{l, n} & \text { in the other cases; }\end{cases}$

$$
U_{j}^{n+1}=\bar{U}_{j}^{n+1}-\Delta_{+} \bar{a}_{j-1 / 2} .
$$




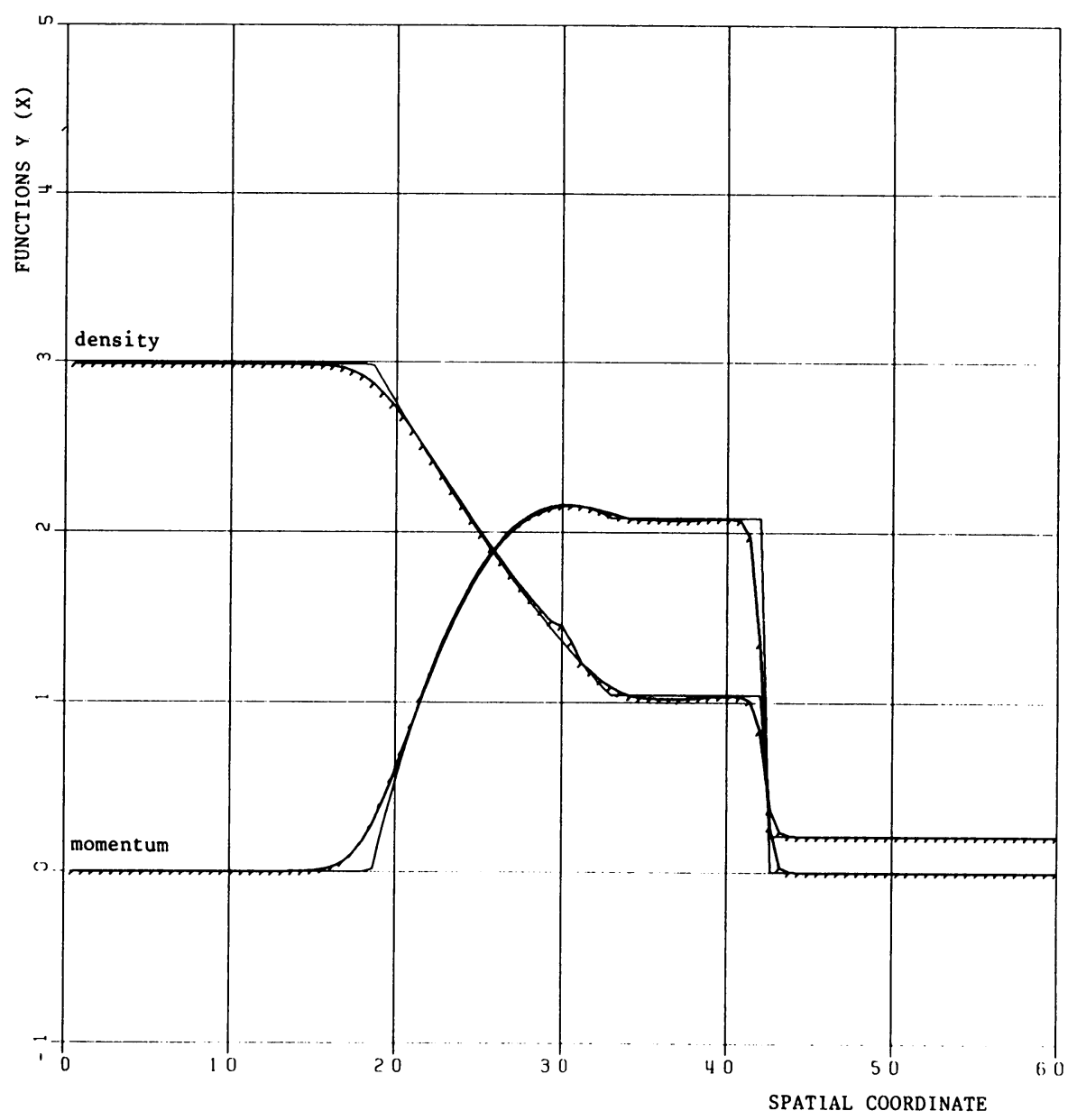

FIGURE 4

L.F. modified scheme $+(3.4)-(3.5)$ correction scheme

\section{Idem Figure 2}

We note that (see Figure 3) the Godunov scheme does not perfectly resolve the rarefaction wave at a sonic point ("dog-leg" phenomenon). As a consequence, we are obliged to use a stricter flux limiter than (3.4), if we want to avoid amplifying this phenomenon by using a higher-order scheme (Figures 5-6).

Figure 4 exhibits the efficiency of our algorithm even for the Lax-Friedrichs modified scheme; the resulting scheme is less accurate than the Godunov scheme plus (5.1)-(5.2), but it is very easy to implement and at low costs. These results show the efficiency of the method compared to a field-by-field flux limiter, which requires diagonalization of $D f$ as in Harten [3]. In Figure 7 we finally present results of 2-dimensional numerical simulation of water waves generated by a landslide in a lake. The basic equations are shallow-water equations (which are similar to Euler isentropic equations). We use the Godunov scheme with the correction algorithm of case 4, and dimensional splitting with the Strang algorithm. Total reflection conditions are used at the boundaries. 
RESULTS FOR $T=.480 E+01$

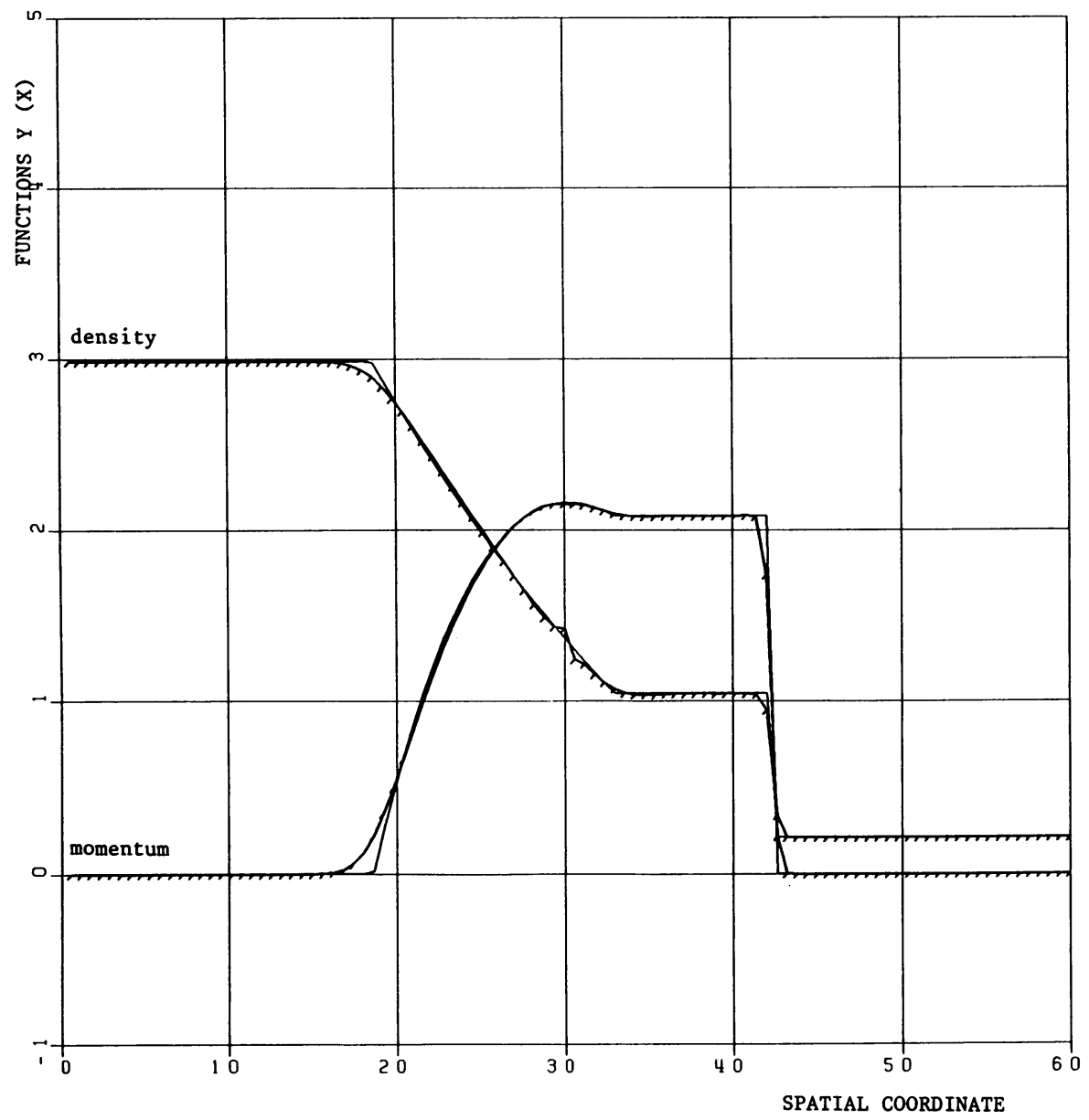

Figure 5

Godunov scheme + (M.C.) correction scheme (5.1)-(5.2)

Idem Figure 3 
RESULTS FOR $T=.480 E+01$

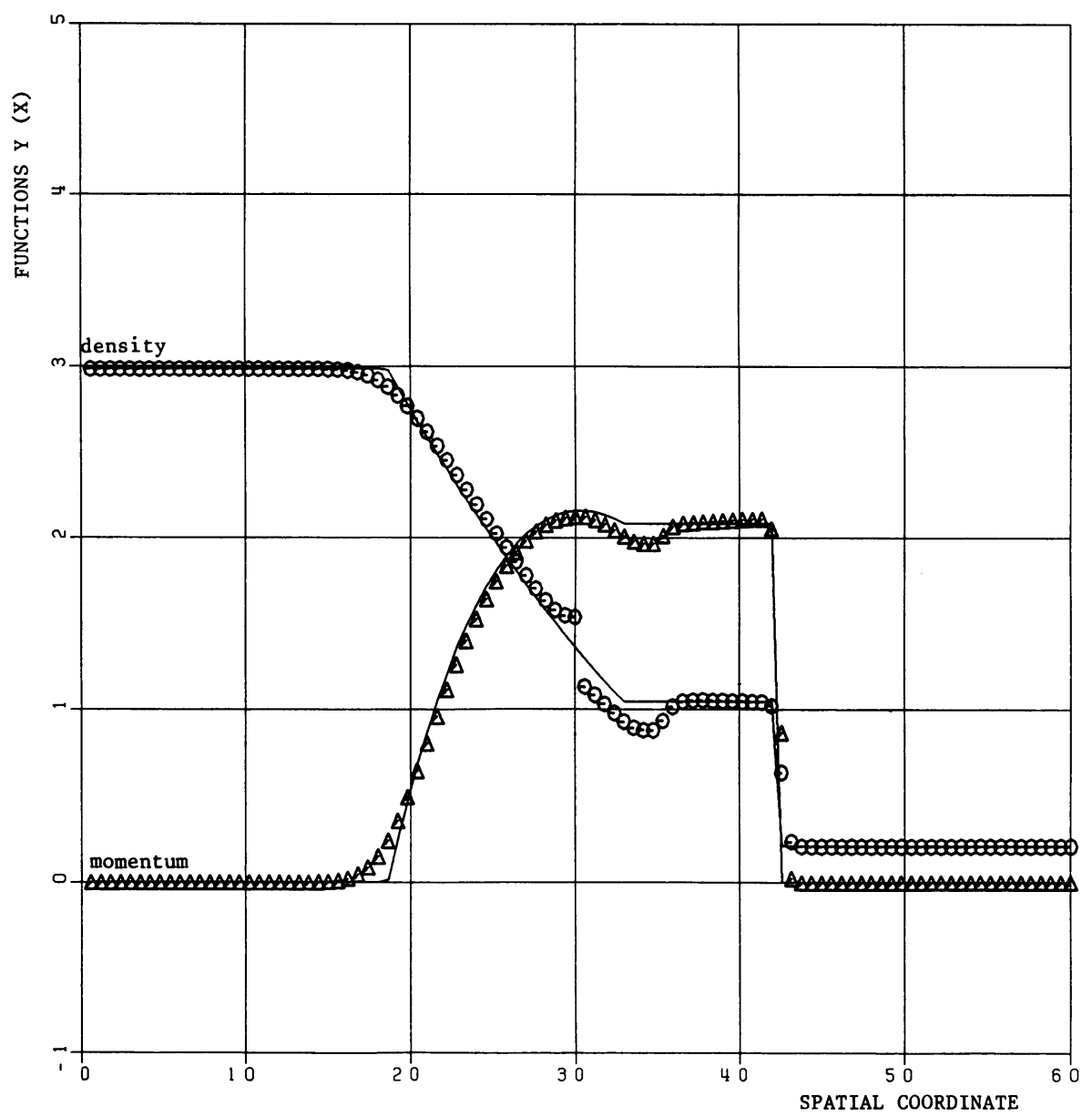

FIGURE 6

Godunov scheme plus correction algorithm (3.4)-(3.5)

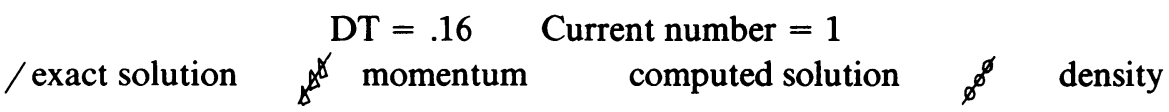


$S U B M E R S I O N \quad 2-D$

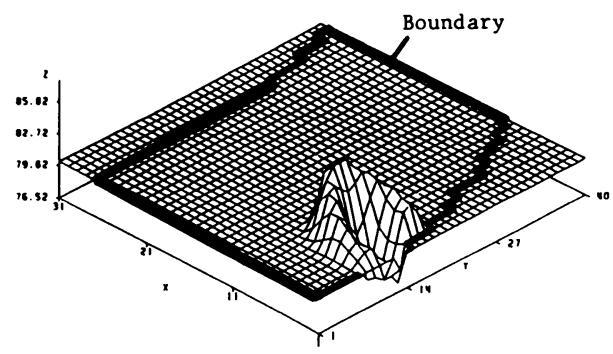

CONTOUR PLOT
$S U B M E R S I O N \underset{\mathrm{T}=17,4 \text { s }}{2-D}$

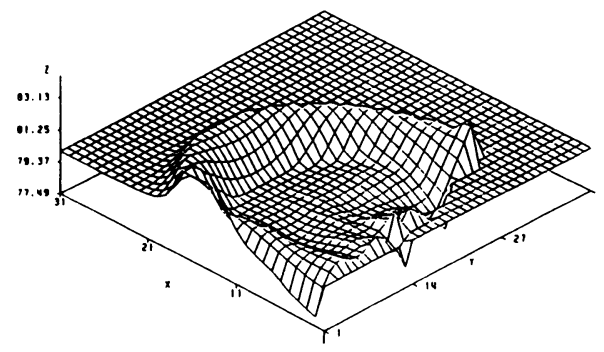

CONTOUR PLOT

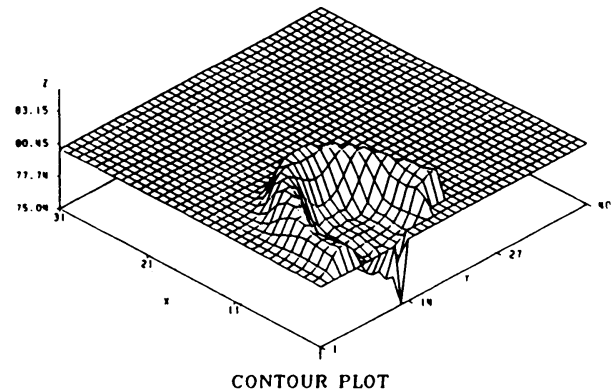

$S U B M E R S I O N \underset{\mathrm{T}=12,6 \mathrm{~s}}{2-D}$

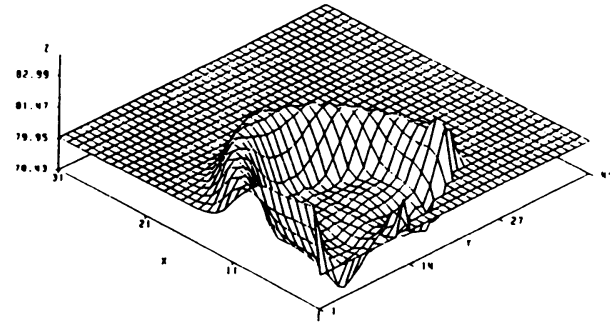

CONTOUR PLOT

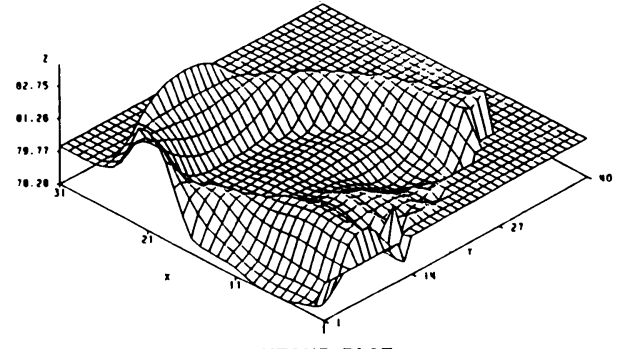

CONTOUR PLOT

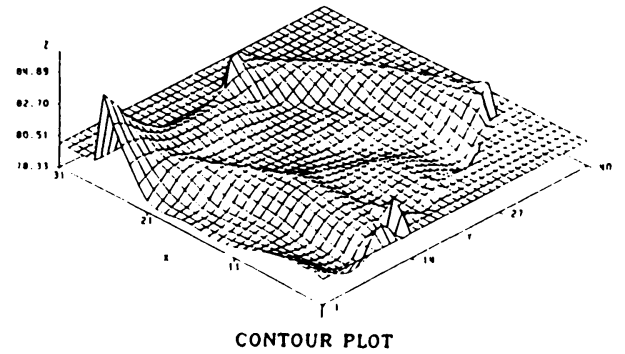

FIGURE 7

Time evolution of water wave generated by landslide, with reflections at boundaries. Grid calculation: $30 \times 40$ 
CEMAGREF

Division Nivologie

Domaine Universitaire

B.P. 76

38402 Saint-Martin-d'Hères, France

1. D. L. Book, J. P. Boris \& K. Hain, "Flux corrected transport. II," J. Comput. Phys., v. 18, 1975, pp. 248-283.

2. R. J. DiPerna, “Uniqueness of solutions to hyperbolic conservation laws," Indiana Univ. Math. J., v. 28,1979 , pp. $137-187$.

3. A. Harten, "High resolution schemes for hyperbolic conservation laws," J. Comput. Phys., v. 49, 1983, pp. 357-393.

4. A. HARTEN \& P. D. LAX, "A random choice finite difference scheme for hyperbolic conservation laws," SIAM J. Numer. Anal., v. 18, 1981, pp. 289-315.

5. A. Harten, P. D. Lax \& B. VAN-Leer, Upstream Differencing and Godunov Type Schemes for Hyperbolic Conservation Laws, ICASE, 82-5.

6. A. Harten, J. M. Hyman \& P. D. LaX, "On finite difference approximations and entropy condition for shocks," Comm. Pure. Appl. Math., v. 29, 1976, pp. 297-322.

7. P. D. LAX, "Shock waves and entropy," in Contributions to Nonlinear Functional Analysis (E. H. Zarantonello, ed.), Academic Press, New York, 1971, pp. 603-634.

8. A. Y. LE Roux, "Numerical stability for some equations of gas dynamics," Math. Comp., v. 37, 1981, pp. 307-320.

9. A. Y. Le Roux \& P. Quesseveur, "Convergence of an antidiffusion Lagrange-Euler scheme for quasilinear equations," SIAM J. Numer. Anal., v. 21, 1984, pp. 985-994.

10. A. MAJDA \& S. OsHer, "A systematic approach for correcting nonlinear instabilities," Numer. Math., v. 30, 1978, pp. 429-452.

11. S. Osher \& S. Chakravarthy, High Resolution Schemes and the Entropy Condition, ICASE 83-49.

12. E. TADMOR, Numerical Viscosity and the Entropy Condition for Conservative Difference Schemes, ICASE 83-20.

13. E. TADMOR, "The large-time behavior of the scalar, genuinely nonlinear Lax-Friedrichs scheme," Math. Comp., v. 43, 1984, pp. 353-368.

14. J. P. VILA, "Simplified Godunov schemes for $2 \times 2$ systems of conservation laws," SIAM J. Numer. Anal., v. 23, 1986, pp. 1173-1192. 